\title{
STRENGTHS AND WIDTHS IN THE FIRST OVERTONE BAND OF HYDROGEN FLUORIDE*
}

\author{
R. E. MEREDITH \\ Willow Run Laboratories, Institute of Science and Technology, The University of Michigan, Ann Arbor \\ 48103, U.S.A.
}

(Received 20 August 1971)

\begin{abstract}
The strengths and self-broadened half widths of 18 lincs of the first overtone band of hydrogen fluoride have been measured using a direct measurement method. The lines were assumed to have the Lorentz or combination Lorentz-Doppler shape.

Matrix elements have been calculated for a dipole moment function of the form $\mu(r)=\sum_{k} M_{k}\left(r-r_{e}\right)^{k}$. Best values of $M_{1}$ and $M_{2}$ were found to be $M_{1}=1.51 \times 10^{-10}$ esu and $M_{2}=-3.74 \times 10^{-3} \mathrm{esu} / \mathrm{cm}$ for a rotating Morse oscillator and $M_{1}=1.52 \times 10^{-10} \mathrm{esu}$ and $M_{2}=-2.15 \times 10^{-3} \mathrm{esu} / \mathrm{cm}$ for the rotating anharmonic oscillator.

Line widths were calculated using the Anderson theory. The first-order effect of the vibrational quantum number on the half widths has been taken into account, and a comparison with measurements on the pure rotation and fundamental bands has been made. The measurements show good agreement with theory in that the half widths tend to decrease with increasing $\Delta v$. Also in agreement with theory, measured values in the $P$ branch are greater than in the $R$ branch for moderate values of $|m|$. As $|m|$ increases and the shorter range forces become more important, the $R$-branch widths tend to become greater than the corresponding lines in the $P$ branch.
\end{abstract}

\section{INTRODUCTION}

KNOWLEDGE of spectral line strengths and widths continues to be of importance in a wide variety of applications. As a partial consequence of this, advances in technology have steadily increased the accuracy with which line parameters are measured. It therefore becomes more and more important to determine the reliability of calculations of strengths and widths in a given band based on measurements in a different band or at different experimental conditions.

The present paper is concerned with two aspects of this problem. These are (1) the extent to which fundamental and overtone line intensities may be predicted on the basis of quadratic electric dipole moment expansion and a rotating oscillator model and (2) the ability of the ANDERSON theory ${ }^{(1)}$ to account for the variations in self-broadened line width within a band and between the pure rotation, fundamental, and first overtone bands. The hydrogen fluoride (HF) molecule has been chosen for this study because extensive measurements have already been made in its pure rotation and fundamental bands ${ }^{(2-4)}$ and because its large dipole moment and small moment of inertia simplify the required calculations considerably. Measurements of strengths and widths in the first overtone band are included in the present study.

* The research was sponsored by the Advanced Research Projects Agency under Contract DAHC15-67-C0062, ARPA Order 236, Amendment 26. 


\section{LINE-STRENGTH AND DIPOLE-MOMENT-FUNCTION CALCULATIONS}

The strength of the vibration-rotation transition $v_{i} J_{i} \rightarrow v_{f} J_{f}$ is related to the matrix element of the electric dipole moment, assuming ideal gas law behavior, as follows

$$
S_{v_{i} v_{f}}(m)=\frac{8 \pi^{3}|m| v \exp \left[T\left(v_{i} J_{i}\right) h c / k T\right]\left|\left\langle v_{i} J_{i}|\mu(r)| v_{f} J_{f}\right\rangle\right|^{2}}{3 h c k T Z} \times 1.0135 \times 10^{6}
$$

where $v$ is the wavenumber of the transition, $T\left(v_{i} J_{i}\right)$ is the term value of the initial level and $Z$ is the vibration-rotation partition function. For $R$ and $P$ branch transitions, $m=J_{f}$ and $m=-J_{i}$, respectively. The matrix elements may be determined experimentally by substituting measured line strengths for $S_{v_{i v}}(m)$. Extrapolation of $\left|\left\langle v_{i} J_{i}|\mu(r)| v_{f} J_{f}\right\rangle\right|^{2}$ to $m=0$ will yield a value of the vibrationless matrix element $\pm\left|\left\langle v_{i}|\mu(r)| v_{f}\right\rangle\right|_{\text {exp }}$.

A function $\mu(r)$ describes the electric dipole moment satisfactorily if suitable wave functions can be used to calculate matrix elements which agree with experimental values. If information is available for the pure rotation, the fundamental, and the first overtone bands, as is the case now for $\mathrm{HF}$, it should be possible to make a three-parameter fit of the dipole moment with experiment. The function $\mu(r)$ may be expanded in a truncated Taylor series as follows

$$
\mu(r)=\sum_{k=0}^{2} M_{k}\left(r-r_{e}\right)^{k}
$$

where $M_{0}, M_{1}$ and $M_{2}$ are the parameters to be determined. Vibrational matrix elements have been computed for this form of $\mu(r)$ for anharmonic oscillator wave functions by HeRMAN and SCHUler ${ }^{(5)}$ and for Morse wave functions by HeAPS and HerZBerg ${ }^{(6)}$. From the results of Herman and Schuler, $M_{1}$ and $M_{2}$ may be determined by solving the set of equations

$$
\begin{aligned}
& \pm\langle 0|\mu(r)| 1\rangle_{\text {exp }}=\left(\frac{M_{1}}{\sqrt{(2 \alpha)}}\right)-\left(\frac{5 b}{\alpha \sqrt{2}}\right) M_{2} \\
& \pm\langle 0|\mu(r)| 2\rangle_{\text {exp }}=\frac{M_{1} b}{\sqrt{(2 \alpha)}}+\frac{M_{2}}{\alpha \sqrt{2}} .
\end{aligned}
$$

Alternatively, from the results of Heaps and Herzberg, $M_{1}$ and $M_{2}$ may be found from the following equations

$$
\begin{aligned}
& \pm\langle 0|\mu(r)| 1\rangle_{\exp }=\frac{M_{1}}{\sqrt{ } 2 \alpha}\left(\frac{\sqrt{ }\left(1-3 x_{e}\right)}{1-2 x_{e}}\right)-\left(\frac{2 x_{e}}{\beta^{2}}\right)\left(\frac{1}{1-2 x_{e}}\right) /\left(\frac{1-3 x_{e}}{x_{e}}\right) \log _{e}\left(1-\frac{5}{2} x_{e}\right) M_{2} \\
& \left. \pm\langle 0|\mu(r)| 2\rangle_{\exp }=\frac{M_{1} r_{e}}{\left(1-3 x_{e}\right)}\right) /\left(\frac{B_{e} x_{e}\left(1-5 x_{e}\right)}{2 \omega_{e}\left(1-2 x_{e}\right)}\right)+\frac{M_{2} r_{e}^{2} B_{e}}{\omega_{e}\left(1-3 x_{e}\right)}\left[\log _{e}\left(1-\frac{7}{2} x_{e}\right)+1\right] /\left(\frac{2\left(1-5 x_{e}\right)}{1-2 x_{e}}\right)^{(4)} .
\end{aligned}
$$

In the above, all spectroscopic constants and Morse parameters retain their usual meaning.

The validity of the $M_{1}$ and $M_{2}$ determined from the relations above may be ascertained by comparing the squares of the matrix elements $\left\langle v_{i} J_{i}|\mu(r)| v_{f} J_{f}\right\rangle$ with those calculated for the rotating anharmonic oscillator and the Pekeris oscillator. These calculations have been performed up to $k=1$ for the anharmonic oscillator by HeRMAN and WALLIS ${ }^{(7)}$ and for the 
Pekeris oscillator by Herman and Rubin ${ }^{(8)}$ and by Herman et al. ${ }^{(9)}$ Their results are expressed as the product of the rotationless matrix element and an $m$ dependent correction factor $F_{v_{i} v_{f}}(m)$, as follows:

$$
\left|\left\langle v_{i} J_{i}|\mu(r)| v_{f} J_{f}\right\rangle\right|^{2}=\left|\left\langle v_{i}|\mu(r)| v_{f}\right\rangle\right|^{2} F_{v_{i} v_{f}}(m) .
$$

Comparison of experimental and theoretical values of the matrix elements will constitute a severe test of the theory and the usefulness of the best fit values of $M_{1}$ and $M_{2}$. A complete discussion and tabulation of the correction factors are given by the above authors.

An extension of the Herman and Wallis calculations to include $M_{2}$ for the rotating anharmonic oscillator has been carried out in a separate report. ${ }^{(10)}$ The details are straightforward and will not be included here. The result for the first overtone band is as follows

$$
\begin{aligned}
F_{o_{2}}(M)= & \left\{1-2 \frac{\gamma^{3 / 2}}{b} m\left[\left(1 \frac{3 \theta}{4}+\frac{\theta b}{2 \sqrt{\gamma}}\right)-\theta \gamma m+\frac{1}{2}\left(2 \frac{\gamma^{1 / 2}}{b}+\frac{15}{4}\right) \gamma^{1 / 2} b(m+5)\right.\right. \\
& \left.\left.+\frac{3}{16} \gamma^{1 / 2} b(2 m-3)\right]+\frac{\gamma^{1 / 2}}{b} \theta_{2}-3 \gamma^{2} \theta_{2} m(m-7)-\frac{3}{2} \frac{\gamma^{5 / 2}}{b} \theta_{2} m\left(m-\frac{3}{2}\right)\right\}^{2}
\end{aligned}
$$

where $\theta=M_{0} / M_{1} r_{e}, \theta_{2}=M_{2} r_{e} / M_{1}$, and $\gamma=2 B_{e} / \omega_{e}$. This expression differs from the Herman and Wallis result only by the inclusion of the terms dependent upon $M_{2}$. One of the latter terms is independent of $m$, and represents the contribution of the electrical anharmonicity. This contribution is expected generally to be small, as the form in which it is written implies, but for certain transitions it may be quite large.

The $M_{2}$ contribution to the Pekeris oscillator has been determined by numerical integration of the integrals involved. The wave functions have been computed using a program written for an IBM 360 computer which was patterned after a program written for a 790 computer by CoOLEY. ${ }^{(11)}$

\section{LINE WIDTH CALCULATIONS}

An extensive description of the Anderson theory and its application to specific molecular encounters has been given by TsaO and CuRnUTTE. ${ }^{(12)}$ A summary of the theory will be given here only to the extent that it will clarify the present calculations and comparisons with experiment. The collision-broadened half width of a vibration-rotation line $v_{i} \rightarrow v_{f}$, $J_{i} \rightarrow J_{f}$ is

$$
\gamma(m)_{v_{i} v_{s}}=\frac{n \bar{v}}{2 \pi c} \sigma
$$

where $\bar{v}$ is the mean relative velocity of an encounter and $n$ is the number of molecules per cubic centimeter at one atmosphere pressure and at temperature $T ; \sigma$ is the real part of a total cross section. It may be written as the sum of partial collision cross sections for each rotation state $J_{2}$ of a perturbing molecule

$$
\sigma=\sum_{J_{2}} \rho_{J_{2}} \sigma\left(J_{2}\right)
$$


where $\rho_{J_{2}}$ weights the contributions of the $\sigma\left(J_{2}\right)$ according to the Boltzmann distribution

$$
\rho_{J_{2}}=\frac{\left(2 J_{2}+1\right) \exp \left(-E_{J_{2}} / k T\right)}{\sum_{J_{2}}\left(2 J_{2}+1\right) \exp \left(\cdot E_{J_{2}} / k T\right)} .
$$

The function $\sigma\left(J_{2}\right)$ is expressed in terms of the development of the interaction in time, as follows

$$
\sigma\left(J_{2}\right)=\int_{0}^{\infty} 2 \pi b S(b) \mathrm{d} b
$$

where $b=b\left(J_{2}\right)$ is the impact parameter and $S(b)$ contains the details of the collision. If it is assumed that the perturbations are due to the interactions involving the electric dipole moment $\mu_{0}$ and the electric quadrupole moment $Q$ of colliding molecules, $S(b)$ may be written as follows

$$
S(b)=\sum_{J_{i}, J_{f}}\left[C_{1} b^{-4} \sum_{l} g_{1 l} f_{1}(k)+C_{2} b^{-6} \sum_{l} g_{2 l} f_{2}(k)+C_{3} b^{-8} \sum_{l} g_{3 l} f_{3}(k)\right]
$$

where

$$
\begin{aligned}
C_{1} & =\frac{4}{9} \frac{\mu_{0}^{4}}{(\hbar \bar{v})^{2}} \\
C_{2} & =\frac{4}{45} \frac{\mu_{0}^{2} Q^{2}}{(\hbar \bar{v})^{2}} \\
C_{3} & =\frac{1}{25} \frac{Q^{4}}{(\bar{h} \bar{v})^{2}} \\
k & =\frac{2 \pi c b}{\bar{v}}\left|(\Delta E)_{s, l}\right|
\end{aligned}
$$

and the $f(k)$ are tabulated in Ref. (12). The $g_{s l}$ are functions of Clebsch-Gordan coefficients. ${ }^{(13)}$ Some particular $g_{s l}$ have been tabulated by BenEDiCT and HeRman. ${ }^{(14)}$ The dipole-dipole, dipole-quadrupole and quadrupole-quadrupole contributions are denoted by $s=1,2$ and 3 respectively. The quantity $(\Delta E)_{s, l}$ is the net rotational nonradiative energy change of the colliding pair of molecules, and the various $\Delta E$ are denoted by the subscript $l$. The energy change may take place while the active molecule is either in its initial level $J_{i}$ or its final level $J_{f}$, in which case

or

$$
\begin{aligned}
& |\Delta E|=\left|E_{J_{i}^{\prime}}-E_{J_{i}}+E_{J_{2}^{\prime}}-E_{J_{2}}\right| \\
& |\Delta E|=\left|E_{J_{f}^{\prime}}-E_{J_{f}}+E_{J_{2}^{\prime}}-E_{J_{2}}\right| .
\end{aligned}
$$

The functions $f(k)$ attain their maximum value near $k=0$ and decrease rapidly to zero as $k$ increases. The cross section $\sigma\left(J_{2}\right)$ therefore is near its largest value for an equal exchange of energy (resonance) between the radiating molecule and its perturber and decreases rapidly as the net energy change increases beyond a critical value. 
The perturbation expansion used to obtain the above expression for $S(b)$ is not valid near $b=0$. Since collisions are expected to be very strong for small $b, S(b)$ is set equal to 1 for all $b$ which cause a strong interaction. That is, the integration of $S(b)$ is taken as follows

$$
\begin{array}{ll}
S(b)=1 & b \leq b_{0} \\
S(b)<1 & b>b_{0} .
\end{array}
$$

This is the approximation number 2 of ANDERSON. ${ }^{(1)}$ The cross section for $J_{2}$ is as follows

$$
\sigma\left(J_{2}\right)=\pi b_{0}^{2}\left[1+C_{1} b_{0}^{-4} \sum_{l} g_{1 l} F_{1}\left(k_{0}\right)+C_{2} b_{0}^{-6} \sum_{l} g_{2 l} F_{2}\left(k_{0}\right)+C_{3} b_{0}^{-8} \sum_{l} g_{3 l} F_{3}\left(k_{0}\right)\right]
$$

where the functions $F(k)$ may be found in Ref. (12).

Specific numerical calculations of half widths assuming no change in the vibrational quantum number have been made by BENEDICT and HERMAN. ${ }^{(14)}$ This assumption is quite good as a first-order approximation. However, collisions between like molecules in different vibrational states are expected to involve inexact resonances which are significant. For example, in this theory, if all perturbers are in the level $v=0$, an inexact resonance will occur in half of the events. In each of these instances the absorbing molecule is in its final level. The HF molecule is a case in point. Measurements on HF lines have indicated a dependence of self-broadened widths on vibrational and rotational quantum numbers which deviates noticeably from that predicted assuming identical upper and lower level energy separations. It is therefore of considerable value to extend the calculations of Benedict and Herman to include near-resonant effects. It will be assumed that the dipoledipole interaction is dominant, and that the quadrupole-quadrupole interaction is negligible. These calculations are feasible even without the use of a high-speed computer since only one term in each of the sums in equations (11) and (14) is appreciable for molecules whose moment of inertia is not too large.

In what follows, it will be assumed that the temperature is low enough so all molecules are initially in the state $v=0$, and that the rotational energy of a level $v, J$ is well approximated by

$$
\begin{aligned}
& E_{v J}=B_{v} J(J+1) \\
& E_{v J}=\left[B_{e}-\alpha_{e}\left(v+\frac{1}{2}\right)\right] J(J+1) .
\end{aligned}
$$

The net energy changes during a collision between a perturber in a level $J_{2}$ and an absorbing molecule in both an initial and a final level are, respectively

$$
\begin{aligned}
& |\Delta E|_{i}=\left|B_{0 J_{i}^{\prime}} J_{i}^{\prime}\left(J_{i}^{\prime}+1\right)-B_{0 J_{i}} J_{i}\left(J_{i}+1\right)+B_{0 J_{2}^{\prime}} J_{2}^{\prime}\left(J_{2}^{\prime}+1\right)-B_{0 J_{2}} J_{2}\left(J_{2}+1\right)\right| \\
& |\Delta E|_{f}=\left|B_{v^{\prime} J_{f}^{\prime}} J_{f}^{\prime}\left(J_{f}^{\prime}+1\right)-B_{v J_{f}} J_{f}\left(J_{f}+1\right)+B_{0 J_{2}^{\prime}} J_{2}^{\prime}\left(J_{2}^{\prime}+1\right)-B_{0 J_{2}} J_{2}\left(J_{2}+1\right)\right| .
\end{aligned}
$$

Exact resonances occur for collisions involving molecules in initial levels, whereas nearresonant collisions occur when final levels are involved. To this approximation then, the near-resonant energy is given by some multiple of $B_{v}-B_{0}$. Table 1 gives the net change for the terms for which near resonance may occur in the first two sums of equation (14). This table confirms the statement above regarding the paucity of terms which contribute for molecules having large $B_{v}$. In the case of HF, the smallest non-resonant energy change is approximately $40 \mathrm{~cm}^{-1}$. Because of the rapid decrease of the function $f_{1}\left(k_{0}\right)$ with increase 
TABle 1. Exact and ineXaCt Resonance ENERGIES FOR Dipole-Dipole AND DIPOLE-QUADRUPOLE INTERACTIONS

\begin{tabular}{ccccc}
\hline Term label & \multicolumn{4}{c}{ Net change } \\
\hline$(s, l)$ & $\Delta J_{i}$ & $\Delta J_{f}$ & \multicolumn{1}{c}{$\Delta J_{2}$} & $(\Delta E)_{s, l}$ \\
$(1,1)$ & & -1 & 1 & $-2 B_{v} J_{f}+2 B_{0}\left(J_{2}+1\right)$ \\
$(1,2)$ & -1 & & 1 & $2 B_{0}\left(J_{2}-J_{i}+1\right)$ \\
$(1,3)$ & & 1 & -1 & $2 B_{v}\left(J_{f}+1\right)-2 B_{0} J_{2}$ \\
$(1,4)$ & 1 & & -1 & $2 B_{0}\left(J_{i}+1-J_{2}\right)$ \\
$(1,5)$ & & -1 & -1 & $-2 B_{v} J_{f}-2 B_{0} J_{2}$ \\
$(1,6)$ & -1 & & -1 & $-2 B_{0}\left(J_{i}+J_{2}\right)$ \\
$(1,7)$ & & 1 & 1 & $2 B_{v}\left(J_{f}+1\right)+2 B_{0}\left(J_{2}+1\right)$ \\
$(1,8)$ & 1 & & 1 & $2 B_{0}\left(J_{i}+J_{2}+2\right)$ \\
$(2,1)$ & & -2 & 1 & $-2 B_{v}\left(2 J_{f}-1\right)+2 B_{0}\left(J_{2}+1\right)$ \\
$(2,2)$ & -2 & & 1 & $-2 B_{0}\left(2 J_{i}-J_{2}-2\right)$ \\
$(2,3)$ & & 1 & -2 & $2 B_{v}\left(J_{f}+1\right)-2 B_{0}\left(2 J_{2}-1\right)$ \\
$(2,4)$ & 1 & & -2 & $2 B_{0}\left(J_{i}-2 J_{2}+2\right)$ \\
$(2,5)$ & & -1 & 2 & $-2 B_{v} J_{f}+2 B_{0}\left(2 J_{2}+3\right)$ \\
$(2,6)$ & -1 & & 2 & $2 B_{0}\left(2 J_{2}-J_{i}+3\right)$ \\
$(2,7)$ & & 2 & -1 & $2 B_{v}\left(2 J_{f}+3\right)-2 B_{0} J_{2}$ \\
$(2,8)$ & 2 & & -1 & $2 B_{0}\left(2 J_{i}-J_{2}+3\right)$ \\
\hline
\end{tabular}

in $k_{0}$, seven of the eight terms are completely negligible for a given $J_{2}$ in the case of the dipole-dipole interaction. The dipole-dipole contributions to $b_{0}\left(J_{2}\right)$ as determined from equation (11) are given in Table 2.

The shorter range dipole-quadrupole interaction will contribute appreciably to those half widths for which the Boltzmann distribution diminishes the importance of the dipole dipole terms. Of the 24 possible contributions, only the second 4 are appreciable. Their contributions to $b_{0}\left(J_{2}\right)$ are given in Table 2 also.

Table 2 emphasizes the $m$ dependence of the collision radii $b_{0}\left(J_{2}\right)$. For example, in the overtone band, a $J_{2}=3$ perturber contributes to half widths for only eight absorber levels: the initial levels of $R(4)$ and $P(4), R(2)$ and $P(2), R(9)$ and $P(9)$, and $R(0)$, and the final levels of $R(3)$ and $P(5), R(1)$ and $P(3), R(8)$ and $P(10)$, and $P(1)$, with radii 9.08, 10.10, 5.76 and $7.26 \AA$, respectively. The table also confirms that in no instance does a particular level receive more than one contribution for a given $J_{2}$. Strictly speaking, there are two additional contributions which originate in the $(2,1)$ and $(2,2)$ terms. These have been omitted since they are several times smaller than the other dipole-quadrupole terms and their inclusion would complicate the calculations considerably. The decrease in the $b_{0}\left(J_{2}\right)$ with increase in $J_{i}$ or $J_{f}$ is due to the decrease of $g_{s l}$ to a constant value in the case of the pure rotation band and also to a more significant inexact resonance for the fundamental and overtone bands. The change in $b_{0}\left(J_{2}\right)$ with $\Delta v$ follows the form of the functions $f_{1}(k)$ and $f_{2}(k)$.

It is not practical to include the quadrupole-quadrupole interaction in the present calculations. Instead, the contribution of the shorter range forces will be inferred by subtracting the calculated widths from experimental values. The half widths $\gamma_{B B}$ obtained in this manner are essentially the "billiard ball" contributions of the so-called resonant dipole-billiard ball model. ${ }^{(15)}$ The present version of this model represents the half width 
TABLE 2. RADII FOR HF-HF COLLISIONS

\begin{tabular}{|c|c|c|c|c|c|c|}
\hline \multirow{2}{*}{$\begin{array}{c}\text { Term label } \\
(s, l) \\
s=1, \text { dipole-dipole } \\
s=2 \text {, dipole-quadrupole }\end{array}$} & \multirow{2}{*}{$J_{i}$ or $J_{f}$} & \multirow{2}{*}{$J_{2}$} & \multicolumn{4}{|c|}{$\begin{array}{c}b_{0}\left(J_{2}\right) \\
(\AA)\end{array}$} \\
\hline & & & \multicolumn{4}{|c|}{$\left.\left.\left.\left.\begin{array}{r}v_{i}=0 \\
v_{f}=0\end{array}\right\} a \begin{array}{c}v_{i}=0 \\
v_{f}=1\end{array}\right\} \begin{array}{c}v_{i}=0 \\
v_{f}=2\end{array}\right\} \begin{array}{c}v_{i}=0 \\
v_{f}=0\end{array}\right\}$} \\
\hline \multirow[t]{10}{*}{$(1,2)$ or $(1,1)^{c}$} & 0 & - & & & & \\
\hline & 1 & 0 & 11.62 & 11.82 & 12.06 & 12.21 \\
\hline & 2 & 1 & 10.99 & 11.40 & 10.98 & 11.55 \\
\hline & 3 & 2 & 10.89 & 11.20 & 10.10 & 11.44 \\
\hline & 4 & 3 & 10.86 & 10.84 & 9.08 & 11.41 \\
\hline & 5 & 4 & 10.84 & 10.40 & 8.30 & 11.39 \\
\hline & 6 & 5 & 10.83 & 9.98 & 7.64 & 11.38 \\
\hline & 7 & 6 & 10.83 & 9.50 & 7.09 & 11.38 \\
\hline & 8 & 7 & 10.83 & 9.07 & 6.62 & 11.38 \\
\hline & 9 & 8 & 10.83 & 8.65 & 6.21 & 11.38 \\
\hline \multirow[t]{9}{*}{$(1,4)$ or $(1,3)^{c}$} & 0 & 1 & 11.62 & 11.82 & 12.06 & 12.21 \\
\hline & 1 & 2 & 10.99 & 11.40 & 10.98 & 11.55 \\
\hline & 2 & 3 & 10.89 & 11.20 & 10.10 & 11.44 \\
\hline & 3 & 4 & 10.86 & 10.84 & 9.08 & 11.41 \\
\hline & 4 & 5 & 10.84 & 10.40 & 8.30 & 11.39 \\
\hline & 5 & 6 & 10.83 & 9.98 & 7.64 & 11.38 \\
\hline & 6 & 7 & 10.83 & 9.50 & 7.09 & 11.38 \\
\hline & 7 & 8 & 10.38 & 9.07 & 6.62 & 11.38 \\
\hline & 8 & 9 & 10.83 & 8.65 & 6.21 & 11.38 \\
\hline \multirow[t]{4}{*}{$(2,6)$ or $(2,5)^{d}$} & 3 & 0 & 7.00 & 7.06 & 7.26 & 8.12 \\
\hline & 5 & 1 & 6.49 & 6.66 & 6.66 & 6.82 \\
\hline & 7 & 2 & 6.35 & 6.60 & 6.20 & 6.67 \\
\hline & 9 & 3 & 6.29 & 6.51 & 5.76 & 6.61 \\
\hline \multirow[t]{4}{*}{$(2,8)$ or $(2,7)^{d}$} & 0 & 3 & 7.00 & 7.06 & 7.26 & 8.12 \\
\hline & 1 & 5 & 6.49 & 6.66 & 6.66 & 6.82 \\
\hline & 2 & 7 & 6.35 & 6.60 & 6.20 & 6.67 \\
\hline & 3 & 9 & 6.29 & 6.51 & 5.76 & 6.61 \\
\hline
\end{tabular}

${ }^{a} T=373^{\circ} \mathrm{K} ;{ }^{b} T=306^{\circ} \mathrm{K} ;{ }^{c} \mu=1.819 ; Q=0 ;{ }^{d} \mu=0 ; Q=9.1 \times 10^{-24} \mathrm{esu} \mathrm{cm}{ }^{2}$.

as the sum of two terms, as follows

$$
\gamma=a(T)(1-f)+b(T) f
$$

where $T$ is temperature and where

$$
f=\sum_{J_{2}} \rho_{J_{2}}
$$

The sum is taken over all $J_{2}$ which contribute in the exact dipole-dipole and dipolequadrupole calculations described above. The remaining $J_{2}$ give rise to $\gamma_{B B}$

$$
\gamma_{B B}=a(T)(1-f)
$$

where $a$ is proportional to a minimum collision radius representing the short-range forces. From experimental half widths $\gamma_{\exp }, a(T)$ may be measured as follows

$$
a(T)=\frac{1}{1-f}\left[\gamma_{\text {exp }}-b(T) f\right] .
$$




\section{EXPERIMENTAL}

The spectrometer used in this investigation was an adaption of a Jarrell-Ash model 78-400. It is of the Ebert-Fastie design, has a 1.83-m focal length, and accepts an $\mathrm{f} / 10$ beam. The grating drive is the Jarrell-Ash sine-bar drive which is designed to establish a linear relationship between wavelength and angle. The slits were built in the instrument shop of The University of Michigan's physics department. The stainless steel jaws have a $4 \frac{1}{4}$-in. radius of curvature. They open unilaterally; the fixed inside jaw is attached to a steel mount. The outer jaw is mounted at the top of a rectangular frame which has two flexible sides made of phosphor bronze. The bottom of the frame is attached to the mount, and the jaws are opened and closed by the motion of a shaft which presses against the top of the frame. When the slits are in the closed position the frame is flexed to a parallelogram shape, and when they are quite wide the frame has relaxed to the original rectangle shape. The motion of the shaft arises from a differential screw arrangement whereby a 25 -thread/in. screw moves inside a screw with 24 threads per in. Photographs of the slits are shown in Fig. 1.

The light source, $s$, is a Mole-Richardson carbon arc model 2371. The crater is slightly less than $\frac{1}{8} \mathrm{in}$. in size and has an effective temperature of $3800^{\circ} \mathrm{K}$. The crater is magnified to $3.5 \mathrm{~cm}$ at $\mathrm{f} / 20$ by a lens (see Fig. 2). This image is then demagnified by mirror $M_{2}$ to $\mathrm{f} / 10$ at the entrance slit. The $f / 20$ divergence and image provide a convenient location for an absorption cell and a 90 cps chopper. The image at the exit slit undergoes a 10 to 1 reduction in size before striking a detector. This is accomplished by a spherical mirror and both a flat and an on-axis ellipse, $M_{4}$. The complete optical path is shown in Fig. 2 . The entire optical path is either evacuated or flushed when spectral regions having atmospheric absorption are being investigated.

A lead sulfide detector having a sensitive area $2.5 \times 0.2 \mathrm{~mm}$ was used for all measurements. A glass Dewar is used in cooling the detector which is mounted at a side view to the

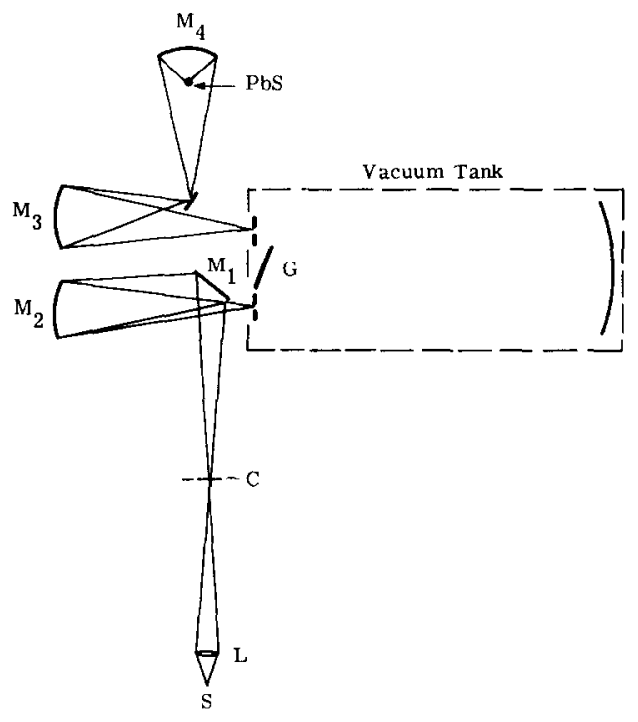

FIG. 2. Diagram of optical path. 